\title{
ERROR ESTIMATION IN THE WEINSTEIN METHOD FOR EIGENVALUES ${ }^{1}$
}

\author{
H. F. WEINBERGER
}

1. Introduction. We are given the eigenvalues $\lambda_{n}$ and eigenvectors $u_{n}$ of a completely continuous positive operator $L$ in a Hilbert space $\mathfrak{S}$. The problem is to determine the eigenvalues of the projection $L^{\prime}$ of $L$ into a subspace $\$ .^{2}$

The method of Weinstein $[1]^{3}$ gives upper bounds for these eigenvalues. If $\left(p_{1}, p_{2}, \cdots\right)$ is any complete set of vectors in the space

$$
\mathfrak{P}=\mathfrak{S} \theta \mathfrak{G},
$$

the $m$ th intermediate problem is to determine the eigenvalues $\lambda_{n}^{(m)}$ of the projection of $L$ into the space

$$
\mathfrak{S} \ominus\left\{p_{1}, \cdots, p_{m}\right\} \text {. }
$$

By the minimax principle, we have

$$
\lambda_{n}^{(m)} \geqq \lambda_{n}^{\prime}
$$

so that the solutions of the $m$ th intermediate problem provide upper bounds for the eigenvalues $\lambda_{n}{ }^{\prime}$. The method of Weinstein consists of explicitly solving the $m$ th intermediate problem in terms of the known eigenvalues and eigenvectors of $L$.

It has been shown (Aronszajn and Weinstein [1]; Aronszajn [1, p. $476 ; 2$, pp. $30-35])$ that for each fixed $n$, and for any complete sequence $\left(p_{1}, p_{2}, \cdots\right)$,

$$
\lim _{m \rightarrow \infty} \lambda_{n}^{(m)}=\lambda_{n}^{\prime}
$$

We are here concerned with the speed of this convergence, that is, with an estimate of the error

$$
\lambda_{n}^{(m)}-\lambda_{n}^{\prime}
$$

Presented to the Society, September 4, 1951; received by the editors December 10, 1951.

1 This work was supported by the Office of Naval Research.

2 We make the convention that the projection of an operator into a subspace is restricted to this subspace, so that the domain of $L^{\prime}$ is $(5$.

3 The formulation of the method in Hilbert space as here presented was given for a special operator by Aronszajn and Weinstein [1], and for the general operator by Aronszajn $[1 ; 2]$. 
2. The error estimate. The upper bounds $\lambda_{n}^{(m)}$, and hence the errors (5), depend upon the sequence $\left(p_{1}, p_{2}, \cdots\right)$. We obtain our error estimate by choosing ${ }^{4}$

$$
p_{n}=\text { projection into } \mathfrak{P} \text { of } u_{n} .
$$

$\left(p_{1}, p_{2}, \cdots\right)$ is clearly complete in $\mathfrak{B}$ since $\left(u_{1}, u_{2}, \cdots\right)$ is complete in $\mathfrak{S}$. We shall show that with the choice (6) of the vectors $p_{n}$,

$$
\lambda_{n}^{(m)}-\lambda_{n}^{\prime} \leqq \lambda_{m+1} \text {. }
$$

Thus, the known eigenvalue $\lambda_{m+1}$ is a uniform estimate of the error (5). Since $L$ is completely continuous, this error estimate can be made arbitrarily small by choosing $m$ sufficiently large.

In proving the inequality (7), we make use of the following inequality given by N. Aronszajn $[1$, p. 476, Corollary I']. For any $m$ th intermediate problem,

$$
\lambda_{n}^{(m)} \leqq \lambda_{n}^{\prime}+\mu,
$$

where $\mu$ is the largest eigenvalue of the projection of $L$ into $\mathfrak{B} \ominus\left\{p_{1}, \cdots, p_{m}\right\}$, that is,

$$
\mu=\max _{p \in \Re \Theta\left\{p_{1}, \cdots, p_{m}\right\}}(L p, p) /(p, p) .
$$

For a general sequence $\left\{p_{n}\right\}, \mu$ is unknown so that (8) does not give an error estimate. However, for the choice (6) of the vectors $p_{n}$ we can estimate $\mu$. We have, for $p$ in $\mathfrak{B} \Theta\left\{p_{1}, \cdots, p_{m}\right\}$,

$$
\left(p, u_{n}\right)=\left(p, p_{n}\right)=0, \quad n=1, \cdots, m .
$$

Then, by the minimax principle,

$$
(L p, p) /(p, p) \leqq \lambda_{m+1} \quad \text { for } p \in \mathfrak{B} \ominus\left\{p_{1}, \cdots, p_{m}\right\}
$$

and so, by (9),

$$
\mu \leqq \lambda_{m+1}
$$

Combining (12) with (8) gives the error estimate (7).

3. Optimum property of the estimate. The error estimate (7) is uniform with regard to the eigenvalues and moreover depends only on the eigenvalues $\lambda_{n}$ of $L$. We now show that it is the best error estimate for the $m$ th intermediate problem having these two properties.

${ }^{4}$ It is easily verified that, if $L$ and $L^{\prime}$ have no common eigenvectors, this sequence is a "suite privilégiée" in the terminology of Weinstein [1].

The explicit construction of the projection in (6) in specific cases will be discussed in another paper. 
Suppose $\mu_{m}$ is such an error estimate. Since it depends only on the eigenvalues of $L$, it must be valid for all projections $L^{\prime}$ of $L$. Take for $L^{\prime}$ the projection in to the space

$$
\left\{u_{r}, u_{r+1}, \cdots\right\} \text {. }
$$

Obviously, we have for this $L^{\prime}$

$$
\lambda_{1}^{\prime}=\lambda_{r}
$$

By the minimax principle, we find that for any $m$ th intermediate problem

$$
\lambda_{1}^{(m)} \geqq \lambda_{m+1}
$$

Subtracting (14) from this gives

$$
\begin{gathered}
\lambda_{1}^{(m)}-\lambda_{1}^{\prime} \geqq \lambda_{m+1}-\lambda_{r} . \\
\mu_{m} \geqq \lambda_{1}^{(m)}-\lambda_{1}^{\prime},
\end{gathered}
$$

by definition of $\mu_{m}$, and hence by (16) and (17),

$$
\mu_{m} \geqq \lambda_{m+1}-\lambda_{r} .
$$

But $r$ is arbitrary and $\lambda_{r} \rightarrow 0$ as $r \rightarrow \infty$. Therefore,

$$
\mu_{m} \geqq \lambda_{m+1},
$$

which proves the optimum property of $\lambda_{m+1}$.

4. Remarks on the Rayleigh-Ritz method. In the Rayleigh-Ritz method as generalized by Aronszajn $[1 ; 2]$, the eigenvalues and eigenfunctions of the projection $L^{\prime}$ of $L$ are assumed to be known and the eigenvalues of $L$ are sought. When $L^{\prime}$ is the projection of $L$ into a finite space, this reduces to the ordinary Rayleigh-Ritz method.

Aronszajn indicated that the same inequality from which (8) is obtained may also lead to an error estimate for the Rayleigh-Ritz method. However, the error term in this case involves the maximum of $(L p, p) /(p, p)$ in a space outside the domain of $L^{\prime}$, so that this estimate cannot be obtained in terms of the eigenvalues of $L^{\prime}$.

In fact, no such estimate is possible. For the eigenvalues of $L^{\prime}$ in no way determine the behavior of $L$ outside the domain of $L^{\prime}$.

However, the situation is different if one knows the eigenvalues and eigenvectors not only of the projection $L^{\prime}$ of $L$ into \& but also of the extension ${ }^{5} L^{\prime \prime}$ of $L$ into a space $\subseteq$ such that

\footnotetext{
- That is, $L^{\prime \prime}$ is completely continuous and $L=L^{\prime \prime}$ in $\mathfrak{S} \cap \mathfrak{S}$.
} 


$$
\mathfrak{S} \supset \mathfrak{B}=\mathfrak{S} \ominus \mathfrak{H} \text {. }
$$

We let the eigenvectors and eigenvalues of $L^{\prime \prime}$ be $u_{n}^{\prime \prime}$ and $\lambda_{n}^{\prime \prime}$. We choose as the $n$th constraint vector $p_{n}$ to be relaxed by the generalized Rayleigh-Ritz method the projection of $u_{n}^{\prime \prime}$ into $\mathfrak{B}$. The generalized Rayleigh-Ritz method enables us to calculate the eigenvalues $\lambda_{n}^{(m)}$ of the projection of $L$ into $\leftrightarrow \oplus\left\{p_{1}, \cdots, p_{m}\right\}$ in terms of the eigenvalues and eigenvectors of $L^{\prime}$. By the definition of eigenvalues, we find that for our choice of $p_{n}$,

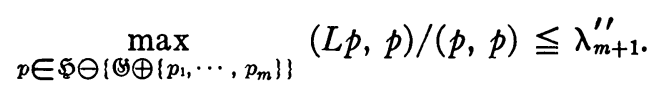

Aronszajn's inequality then gives

$$
\lambda_{n} \leqq \lambda_{n}^{(m)}+\lambda_{m+1}^{\prime \prime}, \quad n=1,2, \cdots .
$$

But the $\lambda_{n}^{(m)}$ are now lower bounds for the $\lambda_{n}$, so that

$$
\lambda_{n}^{(m)} \leqq \lambda_{n} \leqq \lambda_{n}^{(m)}+\lambda_{m+1}^{\prime \prime}
$$

Thus, for the special case where the eigenvalue problems of $L$ in both $\mathbb{B}$ and a space containing $\mathfrak{B}$ are solved, we can obtain the uniform error estimate $\lambda_{m+1}^{\prime \prime}$ for the lower bounds given by the $m$ th intermediate problem of the generalized Rayleigh-Ritz method. This is done by choosing as the $n$th constraint to be released the projection of the eigenvector $u_{n}^{\prime \prime}$ of $L^{\prime \prime}$. If $\mathfrak{S} \supset \mathfrak{S}$, an alternative to the Weinstein method using projections and the Rayleigh-Ritz method is thus obtained.

\section{N. Aronszajn}

\section{BIBLIOGRAPHY}

1. Rayleigh-Ritz and A. Weinstein methods for approximation of eigenvalues. I. Operators in a Hilbert space, Proc. Nat. Acad. Sci. U.S.A. vol. 34 (1948) pp. 474-480, 594-601.

2. Studies in eigenvalue problems. The Rayleigh-Ritz and Weinstein methods for approximation of eigenvalues. I. Operators in a Hilbert space. Technical Report No. 1 (Oklahoma Agricultural and Mechanical College, Stillwater, Oklahoma, 1949).

A. WeInStein

1. Études des spectres des équations aux dérivées partielles de la théorie des plaques élastiques, Mémorial des Sciences Mathématiques vol. 88 (1937).

N. Aronszajn and A. Weinstein

1. On the unified theory of eigenvalues of plates and membranes, Amer. J. Math. vol. 64 (1942) pp. 623-645.

UNIVERSITY OF MARYLAND 